Atmos. Chem. Phys., 10, 2551-2560, 2010

www.atmos-chem-phys.net/10/2551/2010/

(C) Author(s) 2010. This work is distributed under

the Creative Commons Attribution 3.0 License.

\title{
Parameterization of subgrid plume dilution for use in large-scale atmospheric simulations
}

\author{
A. D. Naiman ${ }^{1}$, S. K. Lele ${ }^{1}$, J. T. Wilkerson ${ }^{2}$, and M. Z. Jacobson ${ }^{2}$ \\ ${ }^{1}$ Aeronautics and Astronautics, Stanford University, Stanford, CA, USA \\ ${ }^{2}$ Civil and Environmental Engineering, Stanford University, Stanford, CA, USA \\ Received: 22 October 2009 - Published in Atmos. Chem. Phys. Discuss.: 18 November 2009 \\ Revised: 25 February 2010 - Accepted: 2 March 2010 - Published: 12 March 2010
}

\begin{abstract}
A new model of plume dynamics has been developed for use as a subgrid model of plume dilution in a largescale atmospheric simulation. The model uses mean wind, shear, and diffusion parameters derived from the local largescale variables to advance the plume cross-sectional shape and area in time. Comparisons with a large eddy simulation of aircraft emission plume dynamics, with an analytical solution to the dynamics of a sheared Gaussian plume, and with measurements of aircraft exhaust plume dilution at cruise altitude show good agreement with these previous studies. We argue that the model also provides a reasonable approximation of line-shaped contrail dilution and give an example of how it can be applied in a global climate model.
\end{abstract}

\section{Introduction}

Large-scale atmospheric simulations, such as global climate models, necessarily use coarse spatial grid resolution due to computational cost requirements. Some processes of interest in such models cannot be simulated accurately at the resolved scales because of their nonlinear nature. The chemical reactions and aerosol microphysics that occur in aircraft emission plumes are examples of this problem. Many studies have shown that the chemistry of these plumes proceeds very differently when the emissions are diluted to the grid scale instead of treated as mixing on smaller scales, as happens physically (e.g., Meijer et al., 1997; Petry et al., 1998;

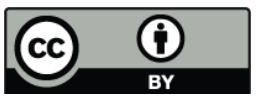

Correspondence to: A. D. Naiman (anaiman@stanford.edu)
Kraabøl et al., 2002). Similarly, the formation of condensation trails (contrails) is a highly visible example of the localized plume processes that would not occur if emitted water vapor and aerosol particles were spread over model grid scales.

Previous studies have treated this problem by means of parameterizations of the nonlinear chemical plume processes (Vohralik et al., 2008; Cariolle et al., 2009). Studies of contrails and the effect of aircraft on cirrus cloud cover have largely neglected subgrid scale processes (e.g., Ponater et al., 2002; Marquart et al., 2003), focusing instead on parameterizing contrail coverage. A recent study developed a parameterization of contrail cirrus based on physical processes including contrail formation, transport, and spreading, tracking coverage, contrail length, and ice mass mixing ratio on the grid scale (Burkhardt and Kärcher, 2009). Burkhardt and Kärcher, in the framework of the ECHAM4 climate model (Roeckner et al., 1996), used a parameterization to determine deposition rates to contrail ice particles, as these clouds exist on a scale of $10 \mathrm{~km}$ compared to a grid spacing of $270 \mathrm{~km}$. They also parameterized the effect of shear on contrail spreading, specifying both a contrail vertical thickness and a spreading constant. Tests in the study did not examine the sensitivity to the specified thickness, but did show significant sensitivity to the spreading parameter.

In order to capture the effect of subgrid scale mixing on nonlinear plume processes, we take a Lagrangian approach in tracking individual aircraft exhaust plumes. We note that a similar approach has recently been taken independently by Schumann (2009). Using a new aircraft emissions inventory that gives individual flight trajectories over a year (Wilkerson et al., 2010) and a global climate model that

Published by Copernicus Publications on behalf of the European Geosciences Union. 


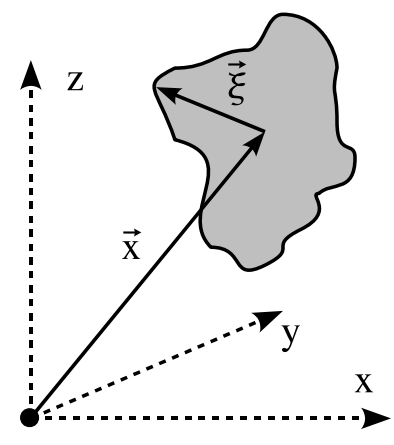

Fig. 1. Schematic of a generalized plume cross-section at a segment end point in the global reference frame.

models aerosol processes on the individual plume level (Jacobson et al., 2010), the parameterizations noted above are exchanged for physical models. In the case of the plume transport and spreading processes, this treatment requires a model that can assess the evolution of the plume location, volume, and shape based on grid scale variables.

This paper presents a model of aircraft plume dilution that is intended to fulfill this role in a large scale atmospheric simulation. It provides prognostic equations for the advancement of the volume and width of a plume based on variables provided by the atmospheric simulation on the grid scale. Although the equations are simple, comparison with a high fidelity model of plume dispersion shows that they are adequate to describe plume dynamics as compared to the level of fidelity of a large scale atmospheric simulation.

Section 2 presents a new model of emission plume dynamics that uses global grid scale variables to advance the plume location, volume, and shape. Section 3 compares the new model with a large eddy simulation of an aircraft exhaust plume, with the analytical solution to the dynamics of a sheared Gaussian plume, and with measurements of aircraft exhaust plume dilution at cruise altitudes. Finally, Sect. 4 argues that the new model also provides a reasonable model of line-shaped contrail dynamics and suggests how it might be used as such in a global climate model.

\section{Subgrid plume model}

In this section, we describe our approach to developing the subgrid plume model (SPM). We describe the basic approximations of the model, derive generalized equations, and then apply them to a specific parameterized plume shape. The resulting model equations can be integrated to analytical solutions with additional minor assumptions, as presented in Sect. 4.3.

\subsection{Approach}

Commercial jet aircraft in cruise emit exhaust that spreads at a much lower rate than the flight speed, resulting in long, slender plumes along their flight path. Our approach in the SPM is to idealize these plumes as high aspect ratio, linear structures with a cross-section that may vary temporally and spatially. Thus, if the aircraft flight trajectory is split into segments, we can represent a corresponding segment of exhaust plume with a length and a cross-section.

The SPM has three goals: to capture the important aspects of aircraft emissions plume evolution under a variety of conditions, to maintain physical properties by obeying conservation laws, and to provide an accurate model of plumes at low computational cost. This third goal is important, as it is the property that allows the SPM to be useful as a subgrid process to track tens of millions of flights during a simulated year in a global model.

To this end, several approximations are made before deriving the SPM equations of motion. First, the SPM treats plumes as tracers of the atmospheric fluid without internal dynamics. Second, the atmospheric disturbances that affect the plume are aggregated into three processes that capture major modes of development: advection due to mean wind, distortion due to wind shear, and dilution due to turbulent mixing. Third, these processes are treated as though they are decoupled. The implications of these approximations with respect to contrails will be examined further in Sect. 4.2.

The three processes treated by the model are applied to the plume segment representation. Advection moves the endpoints of the segment, changing its length. Shear and diffusion act in the plane perpendicular to the segment (due to the slender plume approximation), changing its cross-sectional shape. Quantities needed by the climate model can then be calculated - for example, plume volume is calculated by simply multiplying cross-sectional area by length. This is the approach taken in deriving the SPM equations of motion.

\subsection{General equations of motion}

Figure 1 shows a plume cross-section at a segment endpoint defined by the position vector, $\boldsymbol{x}$, in the global reference frame. The change in the position of the segment endpoint over time is:

$\frac{d x_{i}}{d t}=u_{i}(\boldsymbol{x}, t)$,

where $u_{i}$ is the mean wind component in the $i$ direction. This equation applies advection to the plume segment.

Figure 1 also shows a relative position vector, $\xi$, in a reference frame with its origin at $\boldsymbol{x}$ and the same orientation as the global frame. The change in the position of the piece of plume cross-section at $\xi$ is, to first order,

$\frac{d \xi_{i}}{d t}=\left.\frac{\partial u_{i}}{\partial x_{j}}\right|_{(\boldsymbol{x}, t)} \xi_{j}$. 
This equation represents the kinematic deformation of a material element of the plume and applies the effect of wind shear to the plume cross-section.

The effect of mixing on the plume cross-section is applied using a one-dimensional diffusion equation. The change in the position of the piece of plume cross-section at $\xi$ is:

$\frac{d \xi_{i}}{d t}=\frac{D_{i}}{\xi_{i}}$

where $D_{i}$ is the diffusion coefficient in the $i$ direction. This equation is meant to indicate that the change in each component of $\xi$ is related to the diffusion coefficient in that direction, so there is no sum over $i$ on the right hand side of Eq. (3).

\subsection{SPM equations}

To apply these equations to a model plume, we specify a particular cross-section. The SPM uses an ellipse with three degrees of freedom (two radii and a rotational angle). This choice reflects the results of studies of plumes and contrails under turbulent and shear conditions in the early, vortex phase (Lewellen and Lewellen, 2001; Huebsch and Lewellen, 2006), at later times (Dürbeck and Gerz, 1996; Chlond, 1998), and over longer time frames (Jensen et al., 1998; Unterstrasser and Gierens, 2009). In particular, Schumann et al. (1995) fit a two-dimensional Gaussian plume to their observations of aircraft exhaust plume cross-sections. The SPM is compared to the analytical solution used in that study in Sect. 3.

Since the plume is represented by a high-aspect-ratio segment, shear and diffusion are limited to act on the crosssection only in the plane perpendicular to the segment (the slender plume approximation). Figure 2 shows a schematic of the plume cross-section. The $z$-axis is the same as the global coordinate, while a new horizontal $s$ coordinate is defined as orthogonal to the $z$-axis and in the plane of the crosssection. This figure also defines the three degrees of freedom of the cross-section: $\boldsymbol{a}$, the initially vertical radius of the ellipse; $\boldsymbol{b}$, the initially horizontal radius of the ellipse; and $\theta$, the rotational angle of the ellipse. The angle, $\theta$, is defined as the clockwise angle between the $z$-axis and $\boldsymbol{a}$ and is initially zero.

At cruise altitudes, vertical wind shear dominates the other terms in the velocity gradient tensor, $\partial u_{i} / \partial x_{j}$. Equation (2) can therefore be written for $\boldsymbol{a}$ :

$\frac{d a_{s}}{d t}=\frac{\partial u_{s}}{\partial z} a_{z}$

where $\boldsymbol{a}=a_{s} \hat{s}+a_{z} \hat{z}$ and $u_{s}$ is the projection of the global velocity $\boldsymbol{u}$ onto $\hat{s}$. The ordinary differential equation (ODE) for $\theta$ is derived by relating the components of $\boldsymbol{a}$ to the angle $\theta$ geometrically. Similar manipulations using Eq. (2) result

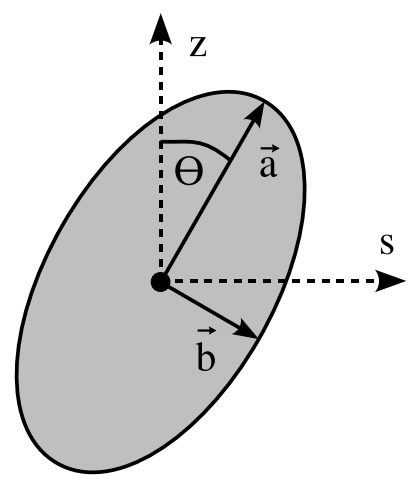

Fig. 2. Definition of geometry for ODE derivation.

in ODEs for the magnitudes of the radii, $a$ and $b$. The set of ODEs describing the effect of shear is:

$\frac{d \theta}{d t}=\frac{\partial u_{s}}{\partial z} \cos ^{2} \theta$,

$\frac{d a}{d t}=a \frac{\partial u_{s}}{\partial z} \sin \theta \cos \theta$,

$\frac{d b}{d t}=-b \frac{\partial u_{s}}{\partial z} \sin \theta \cos \theta$.

Note that these ODEs conserve the cross-sectional area of the plume ellipse.

Diffusion coefficients in the SPM are estimated in the $a$ and $b$ directions as $D_{a}=D_{v} \cos \theta+D_{h} \sin \theta$ and $D_{b}=$ $D_{v} \sin \theta+D_{h} \cos \theta$, where $D_{v}$ and $D_{h}$ are the vertical and horizontal diffusion coefficients, respectively. Applying Eq. (3), the set of ODEs describing the effect of mixing are:

$\frac{d a}{d t}=\frac{D_{a}}{a}$,
$\frac{d b}{d t}=\frac{D_{b}}{b}$.

In summary, Eqs. (1), (5)-(7), and (8)-(9) are advanced in the SPM to determine the location, volume, and shape of a plume segment over time.

\section{Validation comparisons}

\subsection{Comparison with simulations}

An analytical solution to the diffusion of a Gaussian plume in a constant shear flow has been derived (Konopka, 1995) and used in previous studies of plume dynamics (Schumann et al., 1995; Dürbeck and Gerz, 1996). To validate our model, we will compare it with these previous studies of exhaust plumes and show that it produces very similar results. Instead of a Gaussian distribution, the SPM plume represents 

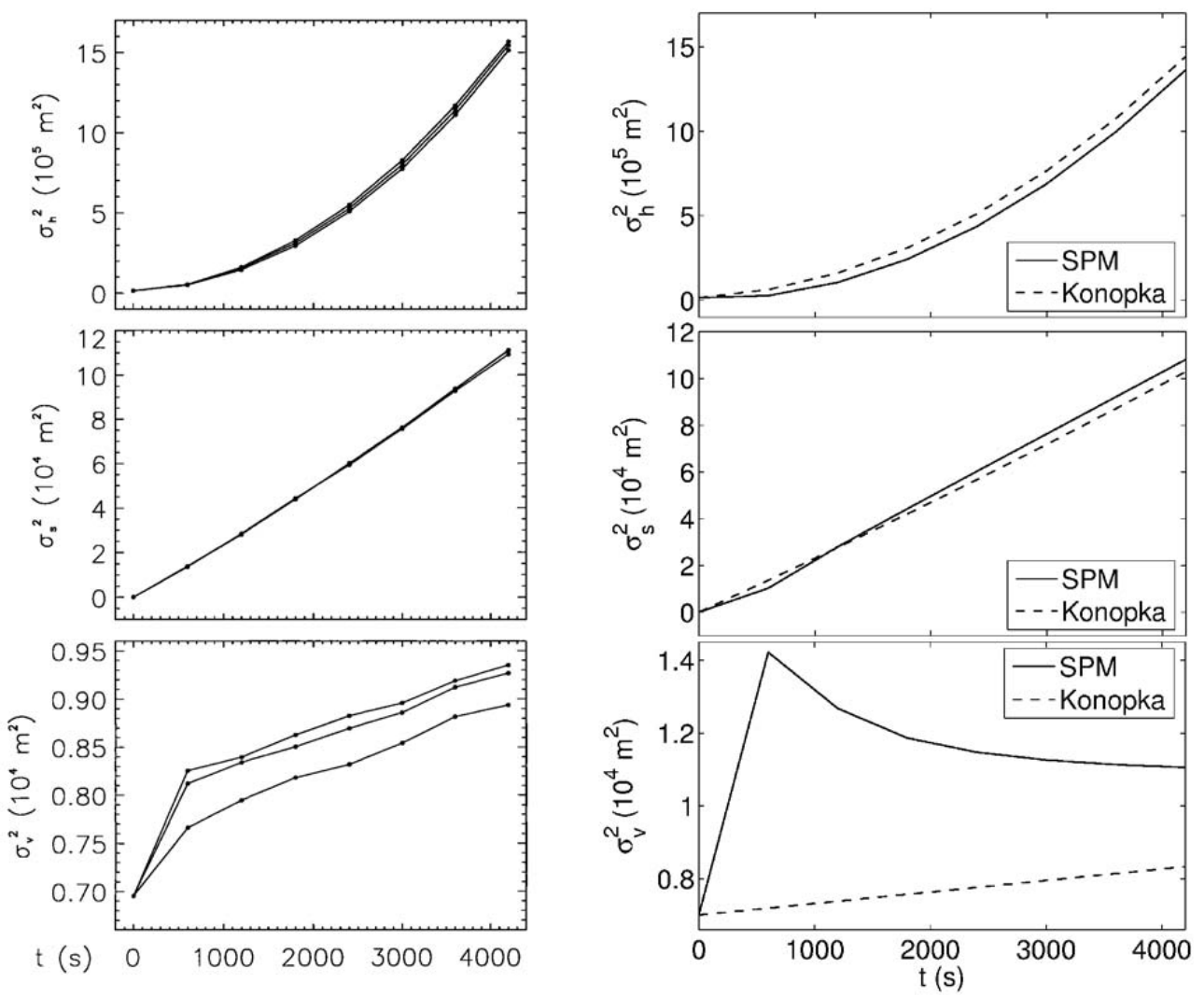

Fig. 3. Horizontal, skewed, and vertical plume variances compared from a computational study and two analytical solutions: Dürbeck and Gerz (1996) LES results (left, their Fig. 7), the SPM equations (right, solid lines), and the Konopka (1995) solution to the diffusion of a Gaussian plume in a uniform shear flow (right, dashed lines). SPM quantities have been converted to plume variances for comparison.

a quasi-uniform distribution of exhaust. Observations and simulations of contrails (e.g., Huebsch and Lewellen, 2006) show that real plumes are somewhere in between these two distributions. In Sect. 4.2 we will further discuss the differences between exhaust plumes and contrails and the significance of the differences between the SPM and the Konopka analytical solution.

Dürbeck and Gerz (1996) conducted a three-dimensional large eddy simulation (LES) study of late (wake-free) aircraft exhaust plume development. The goal of the study was to determine effective diffusion coefficients by fitting twodimensional Gaussians to plume cross-sections computed using LES at different levels of background turbulence and shear. The comparison with the current SPM is thus a simple matter of advancing the SPM equations using the prescribed shear and calculated diffusion parameters from the study.

Figure 3 compares the SPM to the results from the Dürbeck and Gerz study and to the analytical solution to the diffusion of a Gaussian plume in a uniform shear flow (Konopka, 1995). The quanities plotted are $\sigma_{h}^{2}, \sigma_{s}^{2}$, and $\sigma_{v}^{2}$, the horizontal, skewed, and vertical variances of the plume respectively. For the Dürbeck and Gerz plots, these quantities were calculated from the LES results and presented in the reference. Konopka gives analytical expressions for these quantities given a constant vertical shear and diffusion tensor. For comparison, the SPM quantities $a, b$, and $\theta$ have been converted to effective plume variances by projecting the plume ellipse onto the horizontal and vertical axes and solving for the skewed variance:

$$
\begin{aligned}
& \sigma_{v}^{2}=\frac{a^{2}}{4} \cos ^{2} \theta+\frac{b^{2}}{4} \sin ^{2} \theta, \\
& \sigma_{h}^{2}=\frac{a^{2}}{4} \sin ^{2} \theta+\frac{b^{2}}{4} \cos ^{2} \theta, \\
& \sigma_{s}^{2}=\left(\frac{a^{2}}{4}-\frac{b^{2}}{4}\right) \cos \theta \sin \theta .
\end{aligned}
$$

Note that $\sigma_{s}^{2}$ can be either positive or negative, depending on the relative magnitude of $a$ and $b$, but we retain the notation $\sigma_{s}^{2}$ to compare to Dürbeck and Gerz (1996). 

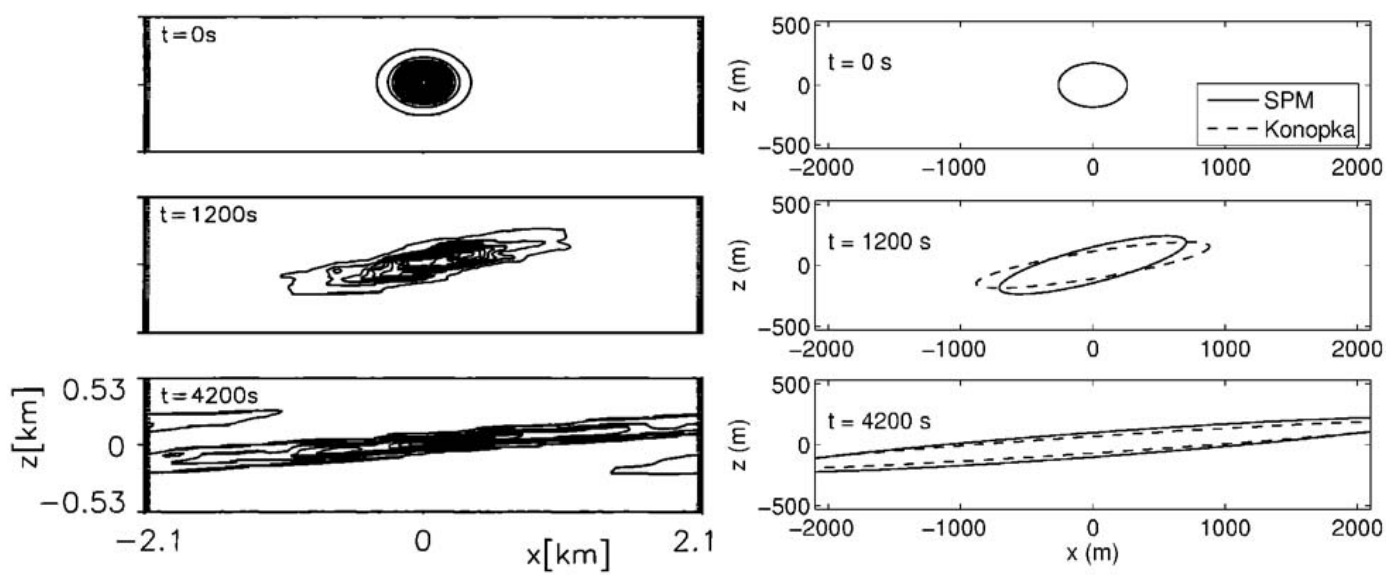

Fig. 4. Comparison of the Dürbeck and Gerz (1996) LES concentration contours (left, from their Fig. 4) with the SPM ellipse and Konopka skewed Gaussian solutions (right). The Konopka Gaussian variances have been converted to effective ellipse radii for comparison.

Figure 3 shows results for the baseline case (case 2 in Dürbeck and Gerz (1996)) using $a_{0}=184 \mathrm{~m}, b_{0}=260 \mathrm{~m}$, $D_{h}=20.0 \mathrm{~m}^{2} / \mathrm{s}, \quad D_{s}=0.75 \mathrm{~m}^{2} / \mathrm{s}, \quad D_{v}=0.158 \mathrm{~m}^{2} / \mathrm{s}$, and $d u_{x} / d z=0.003 \mathrm{~s}^{-1}$. Both the SPM and Konopka analytical solutions match the LES results closely in the growth rates of horizontal and skewed variance. Neither provides a very good match for the growth rate of vertical variance, which, as noted by Dürbeck and Gerz, evolved linearly in the LES over an intial time and a late time, but at two different rates. This quantity also showed the most variation between LES plumes (three plumes were simulated for each case to check the effect of the variability of mixing processes within the flow).

Figure 4 compares contours of exhaust concentration from the Dürbeck and Gerz LES to the SPM and Konopka solutions under the same conditions as noted above. The solutions for all three models are plotted at three times during the seventy minute simulation. Both the SPM and the Konopka model capture the spread of the plume as it is sheared and diffused. These plots illustrate that the difference in the vertical variance development between the models has little effect on the plume extent and horizontal spreading, which is dominated by the vertical shear.

Figure 5 shows the evolution of the plume cross-sectional area normalized by the initial plume area for two of the Dürbeck and Gerz (1996) cases. Case 1 has $d u_{x} / d z=$ $0.001 \mathrm{~s}^{-1}$ and case 4 has $d u_{x} / d z=0.007 \mathrm{~s}^{-1}$, with other parameters as noted for case 2 above. Both the SPM and Konopka analytical solution are plotted for comparison with the Dürbeck and Gerz results (their Fig. 8). Both solutions match the general trend of the LES results, with higher vertical shear causing faster area increase. The SPM solution displays nearly linear behavior (with a small quadratic component) in both cases, whereas the Konopka solution says that the plume area increases as a square root function un- der low shear and a quadratic function under high shear. The LES results showed some of this sensitivity to shear, but were generally more linear than the Konopka solution would suggest. Over the time scale presented by Dürbeck and Gerz, the SPM more closely matches the LES results quantitatively for the high shear case, with the plume area increasing by a factor of eight over the seventy minute simulation.

\subsection{Comparison with observations}

Schumann et al. (1998) calculated the bulk dilution ratio of exhaust plumes using data from more than 70 plume observations at various plume ages from seconds to hours. For the range of ages from less than one second to $10^{4} \mathrm{~s}$, the bulk mean data could be approximated by a curve fit,

$N=7000\left(t / t_{0}\right)^{0.8}$,

where $N$ is the dilution ratio, $t$ is the plume age in seconds, and $t_{0}=1$ is an arbitrary reference scale. Individual observations differed by a factor of 3 from the mean.

Figure 6 shows a comparison of the dilution predicted by the SPM and by the Konopka (1995) analytical solution with this data fit. The SPM and Konopka estimates are shown for the case 1 and case 4 conditions as described in the previous section. Dilution for the models is calculated as the plume area normalized by the initial plume area. The data fit dilution is calculated relative to $t=300 \mathrm{~s}$, a time at which the analytic plume models become applicable, since the wake vortex system of the aircraft can be assumed to have dissipated.

The dilution predicted by the SPM falls well within the factor of 3 scatter in the observational data reported by Schumann et al.. The Konopka model is within the range of scatter for the higher shear case 4, but underpredicts dilution relative to observations for the lower shear case 1 at late times. 

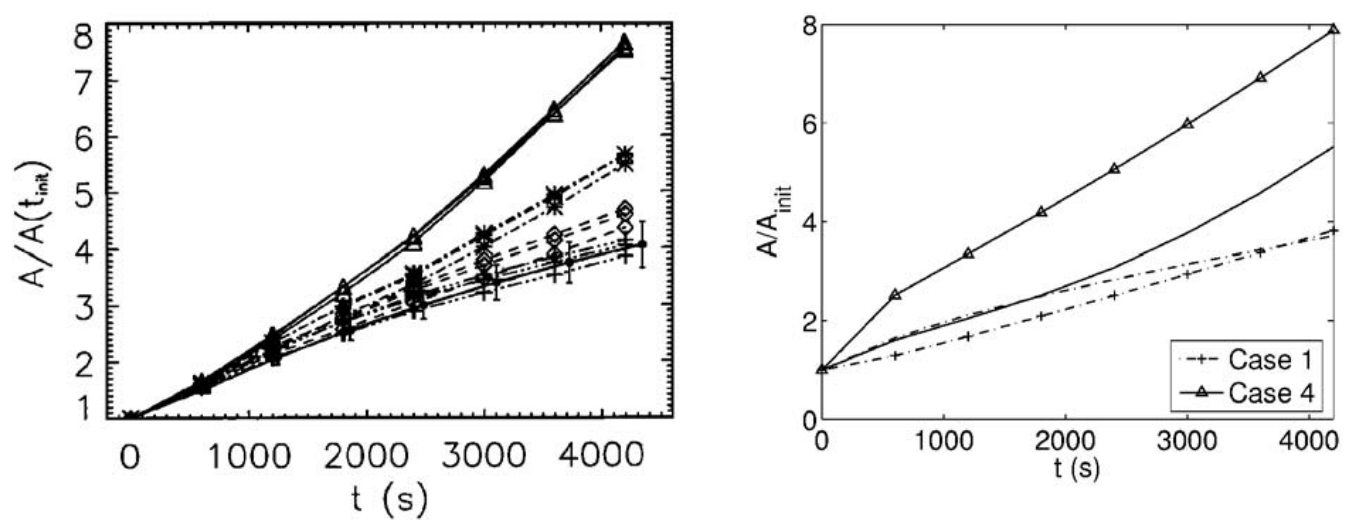

Fig. 5. Normalized plume area compared from a computational study and two analytical solutions: Dürbeck and Gerz (1996) LES results (left, their Fig. 8), the SPM equations (right, lines with symbols), and the Konopka (1995) solution (right, lines with no symbols). The case legend on the right plots matches the original case legend from Dürbeck and Gerz (1996).

\section{Using the subgrid plume model}

The SPM is intended to be used as a subgrid model of aircraft exhaust dynamics, including line-shaped contrails. In this section, we argue that the plume model is suitable for modeling aircraft exhaust and contrail development in the context of a global climate model. We also give an example of how it might be used in such a global model, including analytical solutions to the model equations.

\subsection{Climate model interface}

In order to discuss the implications of the approximations made in deriving the SPM, it is necessary to refer to the usage of the SPM in a particular large-scale atmospheric model, though these arguments could be made for other models of similar scale. The atmospheric model that the SPM was designed for is GATOR-GCMOM, a nested global-to-regional climate model that treats time-dependent gas, aerosol, radiative, dynamical, cloud, land, and ocean processes (Jacobson, 2001a,b, 2002, 2003, 2004). The model is being used to investigate the global impact of aviation on climate (Jacobson et al., 2010) with an emission inventory that specifies individual flight trajectories (Wilkerson et al., 2010).

The climate simulation tracks the volume and shape of emission plumes from individual aircraft over time using SPM segments. For each segment, the simulation uses plume volume to calculate the dilution of plume components for a microphysical model and optical property calculation. Within each segment, particles and their chemical components are tracked over time with a discrete, sizeresolved aerosol-contrail size distribution. Particles grow within each segment by size-resolved coagulation, condensation/evaporation, and ice deposition/sublimation with massconservative and stable numerical methods. The optical properties and shape of each plume segment are used in cal- culations of radiative transfer through plume-occupied portions of the climate simulation grid cells. Segments are tracked individually until they grow to the grid scale or until their water mass concentration is diluted below $10 \mu \mathrm{g} / \mathrm{m}^{3}$. In practice, plumes always become diluted to the threshold water mass concentration before they reach the grid scale size. When this occurs, all components tracked within the plume are conservatively added to the discrete, size-resolved grid-scale aerosol distributions. Once the plume has been added to the grid scale, water vapor and aerosol particles can immediately affect or induce cirrus clouds. Size- and composition-resolved microphysics is calculated for this material at the grid scale, including sedimentation, coagulation, depositional growth, sublimation, and melting. Horizontal and vertical transport are also calculated at the global grid scale. Details of these calculations are described by Jacobson et al. (2010).

When a SPM segment is added to the climate simulation, its initial cross-section is specified based on an estimate of plume sizes at the end of vortex descent from the literature (e.g., Lewellen and Lewellen, 2001). In the first implementation of the SPM, the values $a=120 \mathrm{~m}, b=65 \mathrm{~m}$, and $\theta=0$ are taken as typical for all aircraft in the simulation. This implementation will be refined. An ongoing LES study is developing a database of contrail sensitivities to atmospheric and aircraft parameters. The results from the study will be used to adjust the initial conditions in future implementations.

\subsection{Applicability to contrails}

In Sect. 2.1, we noted several approximations that we used to reduce the subgrid plume model to a more tractable problem. We first treat the plume as a tracer of atmospheric dynamics. Even for a passive plume, this notably neglects the vortex dynamics found in the wake of an aircraft. The vortex wake descends until the Crow instability causes it to break up, 
generally within two minutes after the aircraft passes (Crow and Bate, 1976). This initial descent is essential in spreading the exhaust plume vertically, since the thermal buoyancy of the jet exhaust causes some to detrain from the vortices, leaving a vertical curtain of exhaust (Lewellen and Lewellen, 2001). The time scale of this vortex descent, however, is much smaller than a single time step of the atmospheric simulation $(60 \mathrm{~min})$ and can be accounted for simply by taking this initial descent into account when initializing the plume size (see Sect. 4.1).

In the case of a plume that is not passive, e.g., a contrail that contains significant numbers of ice particles, the tracer approximation also neglects other effects. Line contrail ice particles have been observed to grow to effective particle diameters of $20 \mu \mathrm{m}$ within several hours of contrail formation, with induced cirrus particles up to $200 \mu \mathrm{m}$ observed (e.g., Minnis et al., 1998; Heymsfield et al., 1998; Schröder et al., 2000). This range of particles has terminal settling velocities from 1-10 cm/s (Seinfeld and Pandis, 1998). Sedimentation therefore removes large ice particles from the contrail or contrail-induced cirrus core and increases the vertical extent of the plume directly. In the presence of vertical wind shear, horizontal spread is dominated by the vertical extent of the plume, so sedimentation (to the extent that it occurs) also increases the spread of the plume in the horizontal direction.

Heymsfield et al. (1998) reported that inside the contrail core, where most contrail particles are found, almost all particles remained small (between 1-10 $\mu \mathrm{m}$ diameter). Schröder et al. (2000) likewise described contrails as containing ice crystals with mean diameters in the $1-10 \mu \mathrm{m}$ diameter range. They additionally found young cirrus (i.e., recently formed from contrails) with ice crystals in the 10-20 $\mu$ m diameter range. Using a slip corrected Stokes' Law estimate, the terminal settling velocity of a $20 \mu \mathrm{m}$ diameter particle is approximately $1 \mathrm{~cm} / \mathrm{s}$. Over the lifetime of a long-lived line contrail (e.g., five hours), such a particle would therefore descend approximately $200 \mathrm{~m}$ - on the same order as the initial vertical depth of an SPM segment in the model. The focus of the SPM as used in Jacobson et al. (2010) is to simulate line contrails. While it is true that a small number of particles grow to much larger sizes in line contrails and young cirrus clouds (as reported in the references above), the typical particle is less than $20 \mu \mathrm{m}$ in diameter and does not descend significantly over the typical line contrail lifetime. We therefore neglect the effect of sedimentation on the line contrails modeled using SPM segments. Particles are still allowed to grow to large sizes while contained in the SPM segment if the line contrail is sufficiently long-lived. Once the plume reaches its dilution threshold, all of its aerosol components are added to the grid scale aerosol bins, where sedimentation is accounted for explicitly as a function of particle size and composition.

Another approximation made in deriving the SPM was to aggregate atmospheric disturbances into three processes (advection, vertical shear, and turbulent mixing) and apply them

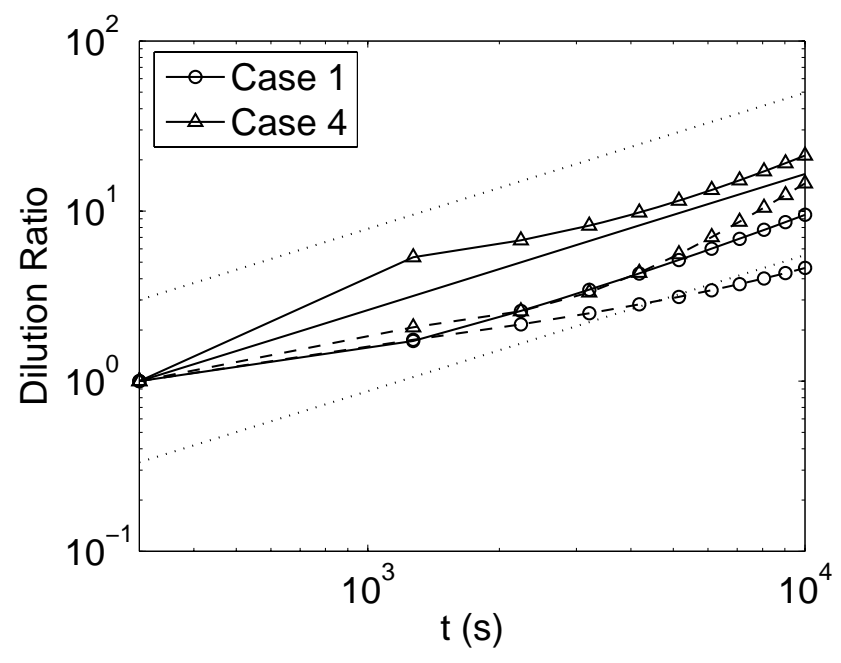

Fig. 6. Dilution ratio plotted against time relative to the plume dilution at $t=300$ seconds. The SPM dilution (solid, symbols) and Konopka (1995) model dilution (dashed, symbols) are plotted for case 1 and case 4 as in Fig. 5. The mean data fit from Schumann et al. (1998) is plotted (solid) along with a factor of 3 scatter range (dotted).

uniformly to a plume segment. In GATOR-GCMOM, as in other global models, subgrid scale variations in parameters that drive these processes in the SPM are captured by diffusion coefficients, which are determined from grid scale shear and stability characteristics. One area of interest is the transition from contrail clouds to cirrus. This transition implies loss of the characteristic linear shape of contrails, which is not accounted for by the SPM. This is related to subgrid perturbations of the velocities that cause vertical shear and turbulence. This transition is accounted for by increasing the diffusion coefficients passed to the the SPM to the levels typically experienced on the aircraft exhaust plume scale (Schumann et al., 1995) as described in Jacobson et al. (2010). Once the contents of the SPM reach a certain level of dilution relative to the ambient atmosphere, they are added to the grid scale, where they can trigger cirrus cloud formation.

Figures 7 and 8 show a final comparison of the SPM and Konopka solutions under the case 1 conditions noted in Sect. 3, advanced over ten hours of simulation time. The two models again match each other closely in terms of plume shape and spread in the horizontal and vertical directions. After ten hours, the SPM predicts the plume area has grown by a factor of 28 compared to the initial area, whereas the Konopka solution predicts a factor of 24 growth. This difference is relatively insignificant in the context of the global atmospheric model. 

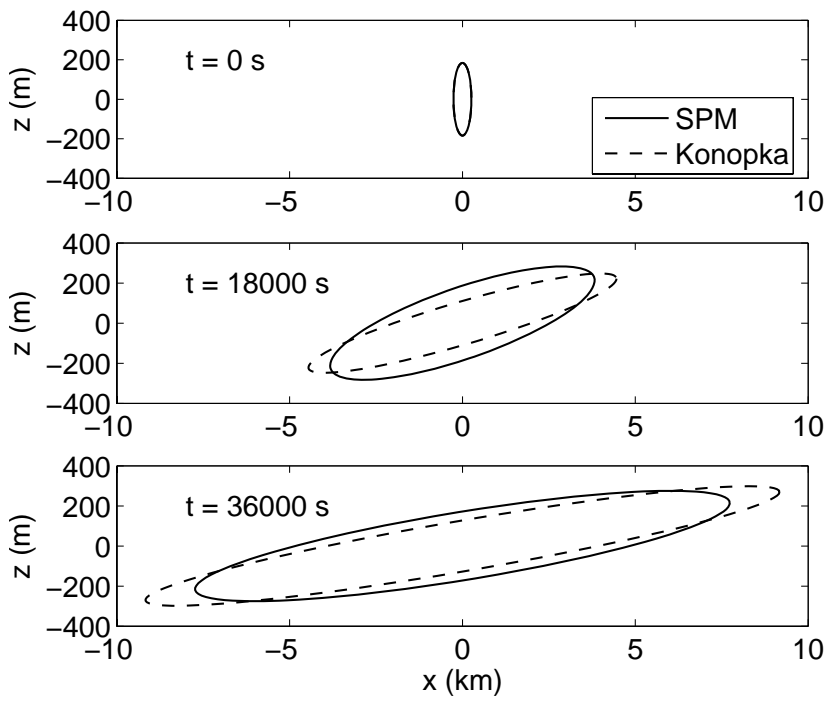

Fig. 7. Comparison of the SPM ellipse and Konopka skewed Gaussian solutions over ten hours of simulation time. The Konopka Gaussian variances have been converted to effective ellipse radii for comparison.

\subsection{Solutions to SPM equations}

This section contains numerical solutions to the ODEs presented in Sect. 2.3 that have been applied inside a global climate simulation (Jacobson et al., 2010). The climate simulation treats the dilution of, microphysics in, and radiation through subgrid line contrail plumes from flights worldwide, but does not currently treat the advection of line contrail position. Although advection of line contrails is not directly treated, contrail components are advected once added to the grid scale following contrail dispersion, where they can induce cirrus cloud formation. In principle, Eq. (1) can be solved using a variety of techniques to directly advect contrail line segments, but the solution to this advection equation should be matched to the model time stepping scheme in which it is implemented.

The remaining SPM equations are solved using the method of operator splitting. First, Eq. (5) is solved analytically by assuming a constant $s=\partial u_{s} / \partial z$ over the time step:

$\theta(t)=\arctan \left(\tan \theta_{0}+s t\right)$,

where $\theta_{0}$ is the value of $\theta$ at the beginning of the time step.

Next, Eqs. (6) and (7) are also solved analytically by again holding $s=\partial u_{s} / \partial z$ constant and using Eq. (14):

$a^{2}(t)=a_{0}^{2}\left(1+s^{2} t^{2} \cos ^{2} \theta_{0}+2 s t \sin \theta_{0} \cos \theta_{0}\right)$,

$b^{2}(t)=\frac{a_{0}^{2} b_{0}^{2}}{a^{2}}$,

where $a_{0}$ and $b_{0}$ are the values of $a$ and $b$ at the beginning of the time step.

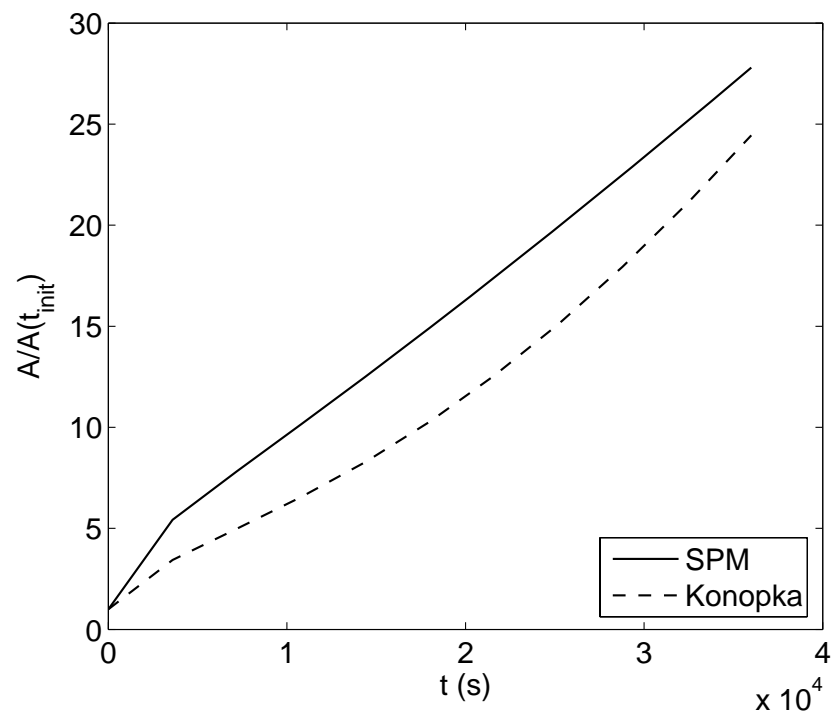

Fig. 8. Comparison of the areas predicted by the SPM and Konopka skewed Gaussian solutions over ten hours of simulation time.

These equations are used in the following discrete form:

$\theta^{n+1}=\arctan \left(\tan \theta^{n}+s \Delta t\right)$,

$\tilde{a}=a^{n}\left(1+s^{2} \Delta t^{2} \cos ^{2} \theta^{n}+2 s \Delta t \sin \theta^{n} \cos \theta^{n}\right)^{1 / 2}$,

$\tilde{b}=\frac{a^{n} b^{n}}{\tilde{a}}$,

where superscripts refer to the time level and $\Delta t$ is the time step.

These intermediate solutions, $\tilde{a}$ and $\tilde{b}$, are then used in the analytic solution of Eqs. (8) and (9):

$a^{n+1}=\sqrt{\tilde{a}^{2}+2 D_{a} \Delta t}$,

$b^{n+1}=\sqrt{\tilde{b}^{2}+2 D_{b} \Delta t}$,

where $D_{a}$ and $D_{b}$ are calculated as noted in Sect. 2.3, using an average value of $\hat{\theta}=\left(\theta^{n+1}+\theta^{n}\right) / 2$ over the time step.

The quantities used by GATOR-GCMOM can be calculated using these values.

$A_{p}^{n+1}=\pi a^{n+1} b^{n+1}$,

$W_{p}^{n+1}=2\left(a^{n+1} \sin \theta^{n+1} \cos r_{x}+b^{n+1} \cos \theta^{n+1} \sin r_{x}\right)$,

where $A_{p}$ is the cross-sectional area of the plume, $W_{p}$ is the projected width of the cross-sectional ellipse, and

$r_{x}=\arctan \left(\frac{b^{n+1}}{a^{n+1} \tan \theta^{n+1}}\right)$.

Multiplying $A_{p}$ by the length of the plume segment gives the plume volume and multiplying $W_{p}$ by the length of the plume segment gives the top-view area of the plume, or the projected area of the plume onto the ground. 


\section{Conclusions}

We have presented a new model of aircraft exhaust plume dilution that is intended to be used as a subgrid scale model within a large scale atmospheric simulation. It provides prognostic equations for the evolution of individual exhaust plumes based on parameters provided by the large scale simulation. Although the equations and their analytical solution are simple to implement, the model shows good agreement with the results of high fidelity, three-dimensional simulations of exhaust plume development.

The model presented has been used as a plume dilution model within a large scale atmospheric simulation, which simulates the evolution of emissions from individual aircraft. Specifically, the dilution has been applied to the aerosol microphysical model to compute the formation and persistence of line-shaped aircraft contrails within the atmospheric simulation. In the future, this model could be used to model other processes, such as dilution for plume chemistry calculations, and for other stationary and moving point sources of emissions. The simplicity of this model makes it a good candidate for such future work, with a low computational cost that would allow it to be used in the Lagrangian tracking of exhaust plumes from many such sources within a large simulation.

Acknowledgements. Thanks to U. Schumann, our referees, and M. Gupta for their helpful comments. This work was supported by the Partnership for AiR Transportation Noise and Emissions Reduction (PARTNER) and the Federal Aviation Administration (FAA) under award number DTFAWA-05-D=0006. Any opinions, findings, and conclusions or recommendations expressed in this material are those of the authors and do not necessarily reflect the views of PARTNER or the FAA. This research is also supported in part by the National Science Foundation through TeraGrid resources provided by LONI and NCSA.

Edited by: S. Galmarini

\section{References}

Burkhardt, U. and Kärcher, B.: Process based simulation of contrail cirrus in a global climate model, J. Geophys. Res., 114, D16201, doi:10.1029/2008JD011491, 2009.

Cariolle, D., Caro, D., Paoli, R., Hauglustaine, D. A., Cuénot, B., Cozic, A., and Paugam, R.: Parameterization of plume chemistry into large-scale atmospheric models: Application to aircraft $\mathrm{NO}_{x}$ emissions, J. Geophys. Res., 114, D19302, doi: 10.1029/2009JD011873, 2009.

Chlond, A.: Large-Eddy Simulation of Contrails, J. Atmos. Sci., 55, 796-819, doi:10.1175/1520-0469(1998)055〈0796:LESOC〉 2.0.CO;2, 1998.

Crow, S. C. and Bate, Jr., E. R.: Lifespan of Trailing Vortices in a Turbulent Atmosphere, J. Aircraft, 13, 476-482, 1976.

Dürbeck, T. and Gerz, T.: Dispersion of aircraft exhausts in the free atmosphere, J. Geophys. Res., 101, 26007-26015, 1996.
Heymsfield, A. J., Lawson, R. P., and Sachse, G. W.: Growth of ice crystals in a precipitating contrail, Geophys. Res. Lett., 25, 1335-1338, 1998.

Huebsch, W. W. and Lewellen, D. C.: Sensitivity Study on Contrail Evolution, in: 36th AIAA Fluid Dynamics Conference and Exhibit, AIAA 2006-3749, 2006.

Jacobson, M. Z.: GATOR-GCMM: A global through urban scale air pollution and weather forecast model, 1. Model design and treatment of subgrid soil, vegetation, roads, rooftops, water, sea ice, and snow, J. Geophys. Res., 106, 5385-5402, 2001 a.

Jacobson, M. Z.: GATOR-GCMM: 2. A study of day- and nighttime ozone layers aloft, ozone in national parks, and weather during the SARMAP Field Campaign, J. Geophys. Res., 106, 5403-5420, 2001b.

Jacobson, M. Z.: Analysis of aerosol interactions with numerical techniques for solving coagulation, nucleation, condensation, dissolution, and reversible chemistry among multiple size distributions, J. Geophys. Res., 107(D19), 4366, doi:10.1029/ 2001JD002044, 2002.

Jacobson, M. Z.: Development of mixed-phase clouds from multiple aerosol size distributions and the effect of the clouds on aerosol removal, J. Geophys. Res., 108(D8), 4245, doi:10.1029/ 2002JD002691, 2003.

Jacobson, M. Z.: The climate response of fossil-fuel and biofuel soot, accounting for soothi's feedback to snow and sea ice albedo and emissivity, J. Geophys. Res., 109, D21201, doi: 10.1029/2004JD004945, 2004.

Jacobson, M. Z., Wilkerson, J. T., Naiman, A. D., and Lele, S. K.: The effects of aircraft on climate and pollution. Part I: A model that treats the subgrid evolution of discrete sizeand composition-resolved contrails from all commercial flights worldwide, J. Geophys. Res.-Atmos., in review, 2010.

Jensen, E. J., Ackerman, A. S., Stevens, D. E., Toon, O. B., and Minnis, P.: Spreading and growth of contrails in a sheared environment, J. Geophys. Res., 103, 31557-31567, 1998.

Konopka, P.: Analytical Gaussian Solutions for Anisotropic Diffusion in a Linear Shear Flow, Journal of Non-Equilibrium Thermodynamics, 20, 78-91, 1995.

Kraabøl, A. G., Berntsen, T. K., Sundet, J. K., and Stordal, F.: Impacts of $\mathrm{NO}_{x}$ emissions from subsonic aircraft in a global three-dimensional chemistry transport model including plume processes, J. Geophys. Res., 107(D22), 4655, doi:10.1029/ 2001JD001019, 2002.

Lewellen, D. C. and Lewellen, W. S.: The Effects of Aircraft Wake Dynamics on Contrail Development, J. Atmos. Sci., 58, 390-406, doi:10.1175/1520-0469(2001)058<0390:TEOAWD $\rangle$. 0.CO;2, 2001.

Marquart, S., Ponater, M., Mager, F., and Sausen, R.: Future Development of Contrail Cover, Optical Depth, and Radiative Forcing: Impacts of Increasing Air Traffic and Climate Change, J. Climate, 16, 2890-2904, doi:10.1175/1520-0442(2003)016<2890: FDOCCO $>2.0 . C O ; 2,2003$.

Meijer, E. W., van Velthoven, P. F. J., Wauben, W. M. F., Beck, J. P., and Velders, G. J. M.: The effects of the conversion of nitrogen oxides in aircraft exhaust plumes in global models, Geophys. Res. Lett., 24, 3013-3016, 1997.

Minnis, P., Young, D. F., and Garber, D. P.: Transformation of contrails into cirrus during SUCCESS, Geophys. Res. Lett., 25, 1157-1160, 1998. 
Petry, H., Hendricks, J., Möllhoff, M., Lippert, E., Meier, A., Ebel, A., and Sausen, R.: Chemical conversion of subsonic aircraft emissions in the dispersing plume: Calculation of effective emission indices, J. Geophys. Res., 103, 5759-5772, 1998.

Ponater, M., Marquart, S., and Sausen, R.: Contrails in a comprehensive global climate model: Parameterization and radiative forcing results, J. Geophys. Res., 107(D13), 4164, doi: 10.1029/2001JD000429, 2002.

Roeckner, E., Arpe, K., Bengtsson, L., Christoph, M., Claussen, M., Dümenil, L., Esch, M., Giorgetta, M., Schlese, U., and Schulzweida, U.: The atmosphere general circulation model ECHAM-4: Model description and simulation of present-day climate, Max Planck Institute Meteorological Report 218, Hamburg, Germany, 1996.

Schröder, F., Kärcher, B., Duroure, C., Ström, J., Petzold, A., Gayet, J. F., Strauss, B., Wendling, P., and Borrmann, S.: On the Transition of Contrails into Cirrus Clouds, J. Atmos. Sci., 57, 464-480, doi:10.1175/1520-0469(2000)057〈0464:OTTOCI $\rangle 2.0$. $\mathrm{CO} ; 2,2000$.

Schumann, U.: A contrail cirrus prediction tool, in: International Conference on Transport, Atmosphere, and Climate, Aachen and Maastricht, 22-25 June 2009, http://www.dlr.de/pa/ en/desktopdefault.aspx/tabid-2559/3824_read-19960/, in press, 2009.
Schumann, U., Konopka, P., Baumann, R., Busen, R., Gerz, T., Schlager, H., Schulte, P., and Volkert, H.: Estimate of diffusion parameters of aircraft exhaust plumes near the tropopause from nitric oxide and turbulence measurements, J. Geophys. Res., 100, 14147-14162, 1995.

Schumann, U., Schlager, H., Arnold, F., Baumann, R., Haschberger, P., and Klemm, O.: Dilution of Aircraft Exhaust Plumes at Cruise Altitudes, Atmos. Environ., 32, 3097-3103, doi:10.1016/ S1352-2310(97)00455-X, 1998.

Seinfeld, J. H. and Pandis, S. N.: Atmospheric chemistry and physics: From air pollution to climate change, chap. 8, John Wiley and Sons, New York, NY, 1998.

Unterstrasser, S. and Gierens, K.: Numerical simulations of contrail-to-cirrus transition - Part 1: An extensive parametric study, Atmos. Chem. Phys. Discuss., 9, 14901-14953, 2009, http://www.atmos-chem-phys-discuss.net/9/14901/2009/.

Vohralik, P. F., Randeniya, L. K., Plumb, I. C., and Baughcum, S. L.: Effect of plume processes on aircraft impact, J. Geophys. Res., 113, D05312, doi:10.1029/2007JD008982, 2008.

Wilkerson, J. T., Jacobson, M. Z., Malwitz, A., Balasubramanian, S., Wayson, R., Fleming, G., Naiman, A. D., and Lele, S. $\mathrm{K}$.: Analysis of emission data from global commercial aviation: 2004 and 2006, Atmos. Chem. Phys. Discuss., 10, 2945-2983, 2010 , http://www.atmos-chem-phys-discuss.net/10/2945/2010/. 